\title{
Association of Circulating Brain-Derived Neurotrophic Factor with Cognition among Adult Obese Population
}

\author{
Ravi Babu Katuri', Girwar Singh Gaur ${ }^{1, *}$, Jaya Prakash Sahoo'², Zachariah Bobby ${ }^{3}$, Karthik Shanmugavel ${ }^{1}$ \\ Departments of ${ }^{1}$ Physiology, ${ }^{2}$ Endocrinology, and ${ }^{3}$ Biochemistry, Jawaharlal Institute of Postgraduate Medical Education and Research (JIPMER), Puducherry, India
}

Background: Obesity has a strong association with the risk of developing cognitive impairment and dementia at a later age. Brain-derived neurotrophic factor (BDNF) and its receptor appear to be important components in cognitive function and are also involved in energy homeostasis. The level of circulating BDNF and its association with cognition has yet to be delineated clearly. In this work we studied the association of circulating BDNF with cognition among the adult obese population.

Methods: The study involved 132 healthy participants between 18 and 40 years of age and of both sexes. The participants were categorized into an obesity group $(n=66)$ and a non-obese group $(n=66)$ based on their body mass index (Asian criteria). The level of cognitive performance was assessed by the event-related potentials P300 (ERPs-P300), mini-mental state examination (MMSE), both visual and auditory reaction times (VRT and ART, respectively), and other pen and paper tests related to memory and executive function. Serum BDNF, glycemic and lipid profiles were estimated.

Results: We found significant differences in the ERPs-P300 latency $(P<0.001)$ and amplitude $(P=0.002)$ between the non-obese and obese group. The MMSE score was significantly reduced while VRT $(P=0.005)$ and ART $(P=0.001)$ were larger in the obese group. BDNF levels $(P<0.001)$ were significantly reduced and negatively associated with the obese group. ERPs-P300 latency was negatively associated $(r=-0.674, P=0.001)$ whereas amplitude $(r=0.507, P<0.001)$ was positively associated with the BDNF levels in the adult obese population. Conclusion: We found reduced circulating BDNF levels in obese adults and that lower BDNF levels were strongly associated with cognitive decline in the obese adult population.

Key words: Brain-derived neurotrophic factor, Cognition, Event-related potentials-P300, Mini-mental state examination, Obesity, Reaction time, BDNF receptor
Received October 10, 2020

Reviewed January 26, 2021

Accepted January 26, 2021

*Corresponding author Girwar Singh Gaur

(iD) https://orcid.org/0000-0002-6593-5187 Department of Physiology, Jawaharlal Institute of Postgraduate Medical Education and Research (JIPMER), Dhanwanthari Nagar, Gorimedu Puducherry 605006, India Tel: +91-999-4470395 Fax: +91-413-2272067 E-mail:drgsgaur@yahoo.com

\section{INTRODUCTION}

Obesity has been determined to be a major health burden in both developing and developed countries. ${ }^{1}$ It causes hypertension, diabetes mellitus, dyslipidemia, coronary heart diseases, and cancer. ${ }^{2}$ In addition to the ill effects of obesity on physical well-being, it also leads to the risk of developing mental health problems such as neurodegenerative disorder, dementia, and cognitive decline by impairing neuroplasticity. ${ }^{3}$ Available evidence has documented a strong association of adulthood obesity with dementia and cogni- tive impairments at a later age. ${ }^{4}$ Cognitive assessment in humans has been done by event-related potentials P300 (ERPs-P300) tests and also both pen and paper tests, such as the mini-mental state examination (MMSE), symbol cancellation tests (SCTs), simple reaction time and trail making tests (TMTs).

Cognition and energy balance have been demonstrated to be governed by the hypothalamus. Evidences from animal experiments and human studies indicated that reduced brain-derived neurotrophic factor (BDNF) leads to increased food intake, weight gain, and adiposity. ${ }^{5}$ Further, decreased circulating BDNF levels 
have led to anorexic signals that caused BDNF receptor mutation in animal models. In accordance with these findings, scientific reports have documented restoration of normal food intake behavior, reduced adiposity, insulin resistance, and other metabolic abnormalities by administering BDNF. ${ }^{6}$ These results have led to an understanding of the interrelationship between circulating BDNF and obesity associated with neurological problems. ${ }^{7}$

However, there is no agreement in the scientific literature related to BDNF levels among the obese population. A few studies have reported increased levels of BDNF in obesity, ${ }^{8}$ but others did not. ${ }^{9}$ One study carried out by Hinderberger et al. ${ }^{10}$ reported a lack of association between obesity and circulating BDNF. As a result, the circulating level of BDNF and its association with obesity-related cognition has yet to be delineated clearly. Hence, in the present study, we aim to assess the association between circulating BDNF levels and cognition, anthropometry, and metabolic parameters in the obese adult population.

\section{METHODS}

\section{Study population}

This was a cross-sectional comparative study conducted in the Department of Physiology of Jawaharlal Institute of Postgraduate Medical and Research (JIPMER), Puducherry, India. After obtaining approvals from the scientific advisory committee (JSAC48/ 2017/68) and institute Human Ethics Committee (JIP/IEC/2017/ 0270), 132 South Indian young adults with ages between 18 and 40 years of both sexes were included in the study. Body mass index (BMI) between 25 and $29.9 \mathrm{~kg} / \mathrm{m}^{2}$ as obese group $(\mathrm{n}=66)$ and apparently healthy non-obese adults with BMI between 18.5 and $22.9 \mathrm{~kg} / \mathrm{m}^{2}$ group $(\mathrm{n}=66)$ were recruited. ${ }^{11}$ Known cases of diabetes, hypertension, history of smoking, alcoholism, neurological disorders, psychological disorders, other endocrinology disorders, and participants who were on drugs for any chronic illness were excluded from our study. Written informed consent was obtained from all the participants prior to the commencement of the study.

\section{Anthropometric measurements}

We recorded height in a standing posture with bare feet, using a wall-mounted stadiometer (VM Electronics, Chicago, IL, USA).
Weight was measured with a digital weighing balance to the nearest $0.1 \mathrm{~kg}$. Hip circumference was measured at the level of the highest point of the gluteal region. Waist circumference (WC) was measured at the widest diameter between the xiphoid process of the sternum and the iliac crest. Obesity indices such as waist to hip ratio (WHR) and BMI were calculated. BMI was calculated as the weight in kilograms divided by the square of the height in meters. ${ }^{12}$

\section{Cognitive parameters}

\section{Event-related potentials}

An ERPs measures the executive function (selective attention) of the individuals by using an auditory oddball paradigm..$^{13}$ The ERPs300 were recorded using "Electrophysiology/Electro-myography machine (Nihon Kohden, Saitama, Japan) \& Neuropack MEB 9200/9300 v08-15" software in a sound-proof room (Electrophysiology lab) around 9 AM after a light breakfast. Participants were asked to sit with their eyes closed while stimuli were given to minimize eye movements and blinking. Electrodes filled with paste, according to the International 10-20 system, were attached to the scalp. The active electrode was placed at $\mathrm{Cz}$ (the intersecting point of nasion-inion and inter-tragus midpoint). Reference electrodes were kept at the right and left mastoid processes. A ground electrode was fixed over the glabella. Impedance was maintained below $5 \mathrm{k} \Omega$. Participants were asked to count silently the infrequent target tones, which were presented randomly with a 0.20 probability in a series of the non-target tones and to report the number of target tones at the end of each run. Latencies and amplitudes on each ERP components were measured. The target and non-target tones were presented at a range of 20 and $80 \%$, respectively, binaurally over headphones at $40 \mathrm{~dB}$. Before ERPs-P300 were recorded, the participants completed several trials until they understood the different tones and the requirements of the test. Subsequently, the recordings were made. Trials including artifacts were automatically rejected from the averages. The procedure was stopped after $35 \mathrm{av-}$ eraged target stimuli. Each test was performed twice to demonstrate the consistency of the waveforms. The waveforms were marked as N100, N200, P200, and P300. The peak latencies were observed at 100,150,200, and $300 \mathrm{~ms}$ after the stimulus onset concerning the waveform. The latencies and amplitudes of N100, N200, P200, and P300 were marked. ${ }^{14}$ 


\section{Reaction time}

The reaction time (RT) apparatus consisted of an automated chronoscope with a four-digit display that recorded the response time with an accuracy of 1 millisecond. For all the participants, RT were recorded using the dominant hand of the participant (all of the subjects were right-handed). RT were measured using both auditory and visual modalities.

\section{Auditory reaction time}

In the auditory reaction time (ART) tests, two sound stimuli (click and beep) were given. The participants were asked to ignore the click sound and respond to the beep sound by releasing a button. The "beep" sound is the "memory" stimulus to which the participant responds by releasing the button and the "click" sound is the "distractor" stimulus to which the participant does not respond. Out of the ten recordings, the two lowermost and two uppermost outlying values were excluded, and the other six values were taken to calculate the mean ART in milliseconds. ${ }^{15}$

\section{Visual reaction time}

To determine the visual reaction time (VRT), two stimuli (red and green colored) were given, and the participants were asked to respond to one stimulus (recognition RT). Red and green colors were shown at random intervals. The participants were asked to respond to the red stimulus by releasing a button. At first, a trial was given. Subsequently, ten readings were taken. The two uppermost and lowermost values were excluded, and the other six values were averaged to determine the mean VRT. ${ }^{15}$

\section{Mini-mental state examination}

The MMSE was administered to assess cognitive function for each participant. The MMSE consists of 11 simple questions grouped into seven cognitive domains: orientation to time and place, registration of three words, attention, calculation, recall of three words, language, and visual construction. The total score was 30 , this score provides a picture of an individual's cognitive performance; a score of $<24$ indicated cognitive impairment. ${ }^{16}$

\section{Mini-cognitive assessment questionnaire}

This questionnaire consists of mainly two domains i.e., recalling memory and executive functions. Participants were informed of a name and an address and given two sets of words to memorize and recall when they were asked. The two sets of recalling words were given as four words in one set and three words in another set. After a minimum of four repetitions the participants were asked to remember these two sets of words, the name, and the address. Participants were asked to recall them after some time. The score was given based on free recall ( 2 points) or clue recall (1 point). Executive function was assessed by asking the participants to draw a clock on a piece of paper and mark the stated time with the arrows. The score was assessed based on the drawn clock (1 point) and the correctly marked time ( 1 point). ${ }^{17}$

\section{Symbol cancellation test}

This test was a paper and pencil test done to assess the sustained attention of the participants. The participants were instructed to mark every open circle crossed by a single slanted line as quickly and accurately as possible. Scoring was done based on the time taken to finish the test (in seconds) and also by counting the number of errors (omissions and commissions) done by the participants. ${ }^{18}$

\section{Symbol trail making test}

The symbol trail making test (STMT) was also a paper and pencil test. This test was similar that of classical TMT. The STMT consisted of two parts, A (STMT-A) and B (STMT-B).

\section{STMT-A}

The participants were asked to connect the different-sized dots continuously from the smallest one to the largest one in increasing order. The time taken to perform the task and the number of the errors were recorded.

\section{STMT-B}

The participants were asked to match shaded and faded symbols. The score reflects the total time required to finish the task and the number of mistakes.

\section{Forward digit span test}

This test assessed the immediate verbal memory span. It contained digit sequences ranging from 3 to 8 numbers. In this test, the 
participants were asked to repeat the digit sequences that were read by the examiner. The numbers were read only once and were not repeated. For every digit sequence, two trials were given. The examiner pronounced a list of digit sequences at a rate of approximately one digit per second and participants were required to immediately repeat the list in the same order. If they succeeded, a sequence one digit longer was presented. If they failed, a second sequence of the same length was presented. If participants were successful on the second list, a list one digit longer was given, as before. However, if participants also failed on the second list, the test ended. The length of the digit sequences gradually increased, starting with a sequence of three numbers (e.g., 2, 9, 8) to a maximum of eight numbers (e.g., $1,3,4,9,6,7,5,2)$. The span was established as the length of the longest list recalled correctly. The number of digits recalled reflected the total score. ${ }^{19}$

\section{Reverse digit span test}

The same procedure for the forward digit span test (FDST) was used for the reverse digit span test (RDST). In the RDST, the participants were asked to repeat the digit sequences in the reverse order of what the examiner said. The score was taken as the maximum number of digits that the subjects were able to reverse. ${ }^{19}$

\section{Measurement of biochemical parameters}

We collected $5 \mathrm{~mL}$ of venous blood for biochemical analysis using an aseptic technique. The fasting blood glucose (FBG) level was estimated by the colorimetric method, the enzymatic method with glucose oxidase, and the peroxidase method, and insulin level (Calbiotech Inc., El Cajon, CA, USA) was estimated using the enzyme-linked immunosorbent assay (ELISA) method. Further, we estimated glycated hemoglobin (HbAlc) with high-performance liquid chromatography. We calculated the homeostatic model assessment of insulin resistance (HOMA-IR) for the participants using the formula $(\mathrm{HOMA}-\mathrm{IR}=\mathrm{FBG}(\mathrm{mmol}) \times$ insulin $(\mu \mathrm{IU} / \mathrm{L}) /$ 22.5). ${ }^{20}$ Lipid profile parameters such as total cholesterol (TC), triglycerides (TG), and high-density lipoprotein cholesterol (HDLC) concentrations were measured using a fully automated clinical chemistry analyzer (ChemWell Awareness Technology, Palm City, FL, USA). The concentration of very low-density lipoprotein (VLDL-C) was calculated using Friedewald equation. ${ }^{21}$ The ath- erogenic index $(\mathrm{AI})$ was calculated using the formula: $\mathrm{AI}=(\mathrm{TC}-$ HDL-C)/HDL-C. ${ }^{22}$ We measured the serum BDNF with ELISA, using a Fine Test (Wuhan Fine Biotech Co., Ltd., Wuhan, China), as per the manufacturer guidelines.

\section{Statistical analysis}

The data was analyzed using IBM SPSS version 20 (IBM Corp. Armonk, NY, USA) software. Normality of the data was analyzed using the Kolmogorov-Smirnov test. All the data were presented as mean \pm standard deviation. The level of significance between the groups was tested using an unpaired t-test. The association between BDNF and various parameters was assessed by Pearson's correlation analysis. The $P$-values $<0.05$ were considered statistically significant.

\section{RESULTS}

\section{Comparison of demographical parameters between the non-obese and obese groups}

There was no significant difference caused by age or sex. Obesity indices such as BMI, WHR, body fat\%, and dry lean fat\% were higher while lean fat $\%$ was lower in the obese group than in the non-obese group (Table 1).

Table 1. Comparison of anthropometric parameters and body fat composition between non-obese and obese groups

\begin{tabular}{lccc}
\hline Variable & Non-obese group $(\mathrm{n}=66)$ & Obese group $(\mathrm{n}=66)$ & $P$ \\
\hline Age $(\mathrm{yr})$ & $27.2 \pm 3.85$ & $28.12 \pm 4.34$ & 0.198 \\
Sex & & & 0.594 \\
$\quad$ Male & $25(47.2)$ & $28(52.8)$ & \\
Female & $41(51.9)$ & $38(48.1)$ & \\
Height $(\mathrm{m})$ & $1.65 \pm 0.09$ & $1.68 \pm 0.08$ & 0.579 \\
Body weight $(\mathrm{kg})$ & $58.72 \pm 7.50$ & $77.12 \pm 8.62$ & $<0.001$ \\
BMI $\left(\mathrm{kg} / \mathrm{m}^{2}\right)$ & $21.26 \pm 1.48$ & $27.21 \pm 1.76$ & $<0.001$ \\
WC $(\mathrm{cm})$ & $75.18 \pm 6.34$ & $91.00 \pm 7.49$ & $<0.001$ \\
HC $(\mathrm{cm})$ & $90.76 \pm 6.50$ & $103.22 \pm 7.88$ & $<0.001$ \\
WHR & $0.82 \pm 0.05$ & $0.88 \pm 0.05$ & $<0.001$ \\
Body fat $(\%)$ & $18.80 \pm 7.79$ & $25.19 \pm 8.34$ & $<0.001$ \\
Lean fat $(\%)$ & $78.82 \pm 14.42$ & $71.95 \pm 15.03$ & $<0.001$ \\
Dry lean weight $(\mathrm{kg})$ & $13.10 \pm 3.57$ & $17.91 \pm 3.58$ & $<0.001$ \\
\hline
\end{tabular}

Values are presented as mean \pm standard deviation or number (\%). The mean differences between the groups were analyzed using an unpaired t-test. A $P$-value $<0.05$ considered statistically significant.

$B M I$, body mass index; WC, waist circumference; $H C$, hip circumference; WHR, waist to hip ratio. 
Table 2. Comparison cognitive parameters between non-obese and obese groups

\begin{tabular}{lccr}
\hline Variable & Non-obese group $(\mathrm{n}=66)$ & Obese group $(\mathrm{n}=66)$ & \multicolumn{1}{c}{$P$} \\
\hline ERPs-P300 & & & \\
Latency (ms) & $316.48 \pm 9.13$ & $345.94 \pm 7.53$ & $<0.001$ \\
Amplitude (mV) & $14.53 \pm 6.48$ & $11.45 \pm 4.25$ & 0.002 \\
Reaction time & & & \\
ART (ms) & $0.20 \pm 0.03$ & $0.22 \pm 0.03$ & 0.001 \\
VRT (ms) & $0.23 \pm 0.03$ & $0.25 \pm 0.03$ & 0.005 \\
MMSE score & $29.89 \pm 0.63$ & $27.56 \pm 3.07$ & $<0.001$ \\
Mini-CA0 Score & $20.21 \pm 1.67$ & $17.84 \pm 3.71$ & $<0.001$ \\
SCT & & & \\
Time (sec) & $70.13 \pm 12.50$ & $78.22 \pm 15.69$ & 0.026 \\
No. of errors & $0.15 \pm 0.40$ & $1.03 \pm 1.50$ & $<0.001$ \\
STMT & & & \\
Time (sec) & $45.80 \pm 13.82$ & $48.67 \pm 17.19$ & 0.304 \\
No. of errors & $0.18 \pm 0.49$ & $0.54 \pm 0.94$ & 0.006 \\
Digit span test & & & \\
FDST score & $6.85 \pm 1.11$ & $6.55 \pm 1.12$ & 0.140 \\
RDST score & $6.00 \pm 1.38$ & $5.31 \pm 1.45$ & 0.006 \\
\hline
\end{tabular}

Values are presented as mean \pm standard deviation. The mean difference between the groups were analyzed using unpaired t-test. A $P$-value $<0.05$ considered statistically significant

ERPs-P300, event-related potentials P300; ART, auditory reaction time; VRT, visual reaction time; MMSE, mini-mental state examination; Mini-CA0, mini-cognitive assessment questionnaire; SCT, symbol cancellation test; STMT: symbol trail making test; FDST, forward digit span test; RDST, reverse digit span test.

Table 3. Comparison of the biochemical parameters between non-obese and obese groups

\begin{tabular}{lccc}
\hline Variable & Non-obese group $(\mathrm{n}=66)$ & Obese group $(\mathrm{n}=66)$ & $P$ \\
\hline FBG $(\mathrm{mg} / \mathrm{dL})$ & $76.96 \pm 6.98$ & $84.65 \pm 15.10$ & $<0.001$ \\
HbA1c & $5.2 \pm 0.26$ & $5.4 \pm 0.38$ & $<0.001$ \\
Insulin $(\mu \mathrm{lU} / \mathrm{mL})$ & $7.77 \pm 3.16$ & $13.86 \pm 4.88$ & $<0.001$ \\
HOMA-IR & $1.03 \pm 0.89$ & $1.81 \pm 1.44$ & $<0.001$ \\
TC $(\mathrm{mg} / \mathrm{dL})$ & $134.53 \pm 20.65$ & $173.95 \pm 35.44$ & $<0.001$ \\
TG $(\mathrm{mg} / \mathrm{dL})$ & $112.16 \pm 38.11$ & $156.20 \pm 64.53$ & $<0.001$ \\
HDL-C $(\mathrm{mg} / \mathrm{dL})$ & $46.60 \pm 9.31$ & $37.18 \pm 11.83$ & $<0.001$ \\
LDL-C $(\mathrm{mg} / \mathrm{dL})$ & $69.69 \pm 25.94$ & $112.06 \pm 33.92$ & $<0.001$ \\
VLDL-C $(\mathrm{mg} / \mathrm{dL})$ & $22.44 \pm 7.61$ & $31.73 \pm 12.86$ & $<0.001$ \\
TC/HDL-C & $3.00 \pm 0.78$ & $5.27 \pm 2.42$ & $<0.001$ \\
TG/HDL-C & $2.52 \pm 1.09$ & $4.92 \pm 3.73$ & $<0.001$ \\
LDL-C/HDL-C & $1.56 \pm 0.70$ & $3.29 \pm 1.93$ & $<0.001$ \\
Atherogenic index & $2.00 \pm 0.78$ & $4.27 \pm 2.42$ & $<0.001$ \\
BDNF $(\mathrm{ng} / \mathrm{mL})$ & $84.27 \pm 9.90$ & $63.79 \pm 17.44$ & $<0.001$ \\
\hline
\end{tabular}

Values are presented as mean \pm standard deviation. The mean difference between the groups were analyzed using unpaired t-test. A $P$-value $<0.05$ considered statistically significant.

FBG, fasting blood glucose; HbA1c, glycated hemoglobin; HOMA-IR, homeostatic model assessment of insulin resistance; TC, total cholesterol; TG, triglycerides; HDL-C, highdensity lipoprotein cholesterol; LDL-C, low-density lipoprotein cholesterol; VLDL-C, very low-density lipoprotein cholesterol; BDNF, brain derived neurotrophic factor.

\section{Comparison of cognitive parameters between the obese and non-obese groups}

The mean latencies of ERPs-P300 increased and the amplitude decreased in the obese group. Reaction times like ART and VRT increased significantly in the obese group. The MMSE and mini-cognitive assessment questionnaire (Mini-CAQ) scores were higher in the non-obese group compared to the obese group. The SCT time and the number of errors were smaller in the non-obese group. The STMT time was comparable between the groups but the number of errors was significantly higher in the obese group than in the non-obese group. The RDST score was significantly higher in the non-obese group while the FDST score was comparable (Table 2).

Table 4. Correlation of BDNF with anthropometric, cognitive, glycemic, and lipid profile parameters in non-obese and obese populations

\begin{tabular}{|c|c|c|c|c|}
\hline \multirow{2}{*}{ Variable } & \multicolumn{2}{|c|}{ Non-obese group $(n=66)$} & \multicolumn{2}{|c|}{ Obese group $(n=66)$} \\
\hline & r & $P$ & $r$ & $P$ \\
\hline BMI (kg/m²) & -0.596 & $<0.001$ & -0.658 & $<0.001$ \\
\hline WHR & -0.166 & 0.183 & -0.267 & 0.030 \\
\hline ERPs-P300 latency (ms) & -0.454 & $<0.001$ & -0.674 & $<0.001$ \\
\hline ERPs-P300 amplitude (mV) & 0.028 & 0.822 & 0.507 & $<0.001$ \\
\hline ART (ms) & -0.232 & 0.061 & -0.283 & 0.021 \\
\hline VRT (ms) & -0.232 & 0.061 & -0.524 & $<0.001$ \\
\hline STMT time (sec) & -0.162 & 0.192 & -0.137 & 0.272 \\
\hline No. of errors & 0.148 & 0.234 & 0.141 & 0.259 \\
\hline SCT time (sec) & -0.080 & 0.528 & -0.292 & 0.017 \\
\hline No. of errors & -0.207 & 0.096 & -0.545 & $<0.001$ \\
\hline Mini CAO score & 0.070 & 0.573 & 0.725 & $<0.001$ \\
\hline MMSE score & 0.004 & 0.977 & 0.530 & $<0.001$ \\
\hline FDST & 0.003 & 0.981 & 0.272 & 0.027 \\
\hline RDST & 0.236 & 0.056 & 0.534 & $<0.001$ \\
\hline FBG (mg/dL) & -0.023 & 0.855 & -0.794 & $<0.001$ \\
\hline $\mathrm{HbA1c}$ & -0.221 & 0.145 & -0.577 & $<0.001$ \\
\hline Insulin ( $\mu \mid \mathrm{U} / \mathrm{mL})$ & -0.029 & 0.849 & -0.660 & $<0.001$ \\
\hline HOMA-IR & -0.010 & 0.935 & -0.785 & $<0.001$ \\
\hline $\mathrm{TC}(\mathrm{mg} / \mathrm{dL})$ & -0.018 & 0.888 & -0.619 & $<0.001$ \\
\hline TG (mg/dL) & -0.212 & 0.087 & -0.701 & $<0.001$ \\
\hline $\mathrm{HDL}-\mathrm{C}$ (mg/dL) & 0.232 & 0.060 & 0.573 & $<0.001$ \\
\hline LDL-C (mg/dL) & -0.178 & 0.154 & -0.673 & $<0.001$ \\
\hline Atherogenic index & -0.204 & 0.101 & -0.682 & $<0.001$ \\
\hline
\end{tabular}

Pearson correlation was used. A $P$-value $<0.05$ considered statistically significant. $\mathrm{BDNF}$, brain-derived neurotrophic factor; BMI, body mass index; WHR, waist to hip ratio; ERPs-P300, event-related potentials P300; ART, auditory reaction time; VRT, visual reaction time; STMT, symbol trail making test; SCT, symbol cancellation test; Mini-CAO, mini-cognitive assessment questionnaire; MMSE, mini-mental state examination; FDST, forward digit span test; RDST, reverse digit span test; FBG, fasting blood glucose; $\mathrm{HbA1C}$, glycated hemoglobin; HOMA-IR, homeostatic model assessment of insulin resistance; TC, total cholesterol; TG, triglycerides; HDL-C, high-density lipoprotein cholesterol; LDL-C, low-density lipoprotein cholesterol. 


\section{Comparison of biochemical parameters between non-obese and obese groups}

FBG, HbAlc, fasting insulin, and HOMA-IR were higher in the obese group. The obese group had significantly higher mean values of TC, TG, LDL-C, VLDL-C and lipid derived parameters like AI, TC/HDL-C, TG/HDL-C, and LDL-C/HDL-C. BDNF and HDL-C were significantly lower in the obese group (Table 3 ).

\section{Association of BDNF with different parameters}

BMI and WHR were negatively correlated in the obese group. ERPs-P300 latency was negatively correlated while amplitude was positively correlated in the obese group. RT were negatively corre- lated in the obese group. SCT time and errors were negatively correlated. MMSE, Mini-CAQ, FDST, and RDST scores were positively correlated in the obese group. FBG, $\mathrm{HbAlc}$, insulin, HOMAIR, TC, TG, LDL-C, and AI were negatively correlated while HDL-C was positively correlated in the obese group (Table 4).

Multiple linear regression analyses depicted the individual contribution of obesity indices like BMI, WC, WHR, fat $\%$, TC, and TG to BDNF levels in the non-obese group and the obese group (Table 5). The regression analyses also revealed the individual association of cognitive parameters (MMSE, ERPs-P300, RT, MiniCAQ, DST, and SCT) to BDNF levels in the non-obese and obese groups (Table 6). The serum BDNF levels were higher in females

Table 5. Multiple linear regression analysis of BDNF (as dependable variable) with obesity indices (as independent variables) in non-obese and obese groups

\begin{tabular}{|c|c|c|c|c|c|c|c|c|}
\hline \multirow{3}{*}{$\begin{array}{l}\text { Independent } \\
\text { variable }\end{array}$} & \multicolumn{4}{|c|}{ Non-obese group $(n=66)$} & \multicolumn{4}{|c|}{ Obese group $(n=66)$} \\
\hline & \multirow{2}{*}{$\begin{array}{l}\text { Standardized } \\
\text { coefficient } \beta\end{array}$} & \multicolumn{2}{|c|}{$95 \% \mathrm{Cl}$ of unstandardized $\beta$} & \multirow{2}{*}{$P$} & \multirow{2}{*}{$\begin{array}{l}\text { Standardized } \\
\text { coefficient } \beta\end{array}$} & \multicolumn{2}{|c|}{$95 \% \mathrm{Cl}$ of unstandardized $\beta$} & \multirow{2}{*}{$P$} \\
\hline & & $L B$ & UB & & & LB & UB & \\
\hline BMl & -0.520 & -5.350 & -1.662 & $<0.001$ & -0.423 & -6.721 & -1.768 & 0.001 \\
\hline $\mathrm{WC}(\mathrm{cm})$ & -0.145 & -0.728 & 0.278 & 0.370 & -0.084 & -0.619 & 0.226 & 0.356 \\
\hline WHR & -0.031 & -53.66 & 41.99 & 0.808 & -0.057 & -61.544 & 28.121 & 0.458 \\
\hline Fat $\%$ & 0.058 & -0.195 & 0.344 & 0.583 & 0.185 & 0.013 & 0.773 & 0.043 \\
\hline $\mathrm{TC}(\mathrm{mg} / \mathrm{dL})$ & 0.052 & -0.074 & 0.124 & 0.614 & -0.205 & -0.190 & -0.013 & 0.025 \\
\hline $\mathrm{TG}(\mathrm{mg} / \mathrm{dL})$ & -0.044 & -0.067 & 0.044 & 0.683 & -0.378 & -0.154 & -0.050 & $<0.001$ \\
\hline
\end{tabular}

A $P$-value $<0.05$ considered statistically significant.

BDNF, brain-derived neurotrophic factor; Cl, confidence interval; LB, lower bound; UB, upper bound; BMI, body mass index; WC, waist circumference; WHR, waist to hip ratio; TC, total cholesterol; TG, triglycerides.

Table 6. Multiple linear regression analysis of BDNF (independent variable) with cognitive parameters (as dependable variables) in non-obese and obese groups

\begin{tabular}{|c|c|c|c|c|c|c|c|c|}
\hline \multirow{3}{*}{$\begin{array}{l}\text { Independent } \\
\text { variable }\end{array}$} & \multicolumn{4}{|c|}{ Non-obese group $(n=66)$} & \multicolumn{4}{|c|}{ Obese group $(n=66)$} \\
\hline & \multirow{2}{*}{$\begin{array}{l}\text { Standardized } \\
\text { coefficient } \beta\end{array}$} & \multicolumn{2}{|c|}{$95 \% \mathrm{Cl}$ of unstandardized $\beta$} & \multirow{2}{*}{$P$} & \multirow{2}{*}{$\begin{array}{c}\text { Standardized } \\
\text { coefficient } \beta\end{array}$} & \multicolumn{2}{|c|}{$95 \% \mathrm{Cl}$ of unstandardized $\beta$} & \multirow{2}{*}{$P$} \\
\hline & & LB & UB & & & LB & UB & \\
\hline MMSE & 0.010 & -0.015 & 0.017 & 0.939 & 0.500 & 0.050 & 0.126 & $<0.001$ \\
\hline \multicolumn{9}{|l|}{ ERPs-P300 } \\
\hline Latency (ms) & -0.455 & -0.609 & -0.209 & $<0.001$ & -0.152 & -0.299 & 0.071 & 0.222 \\
\hline Amplitude (mV) & 0.030 & -0.144 & 0.183 & 0.814 & 0.565 & 0.088 & 0.188 & $<0.001$ \\
\hline $\mathrm{ART}(\mathrm{ms})$ & -0.231 & -0.001 & 0.000 & 0.063 & -0.357 & -0.001 & 0.000 & 0.003 \\
\hline VRT (ms) & -0.231 & -0.001 & 0.000 & 0.063 & -0.563 & -0.002 & -0.001 & $<0.001$ \\
\hline Mini-CAO & 0.073 & -0.029 & 0.055 & 0.549 & 0.706 & 0.113 & 0.188 & $<0.001$ \\
\hline FDST & 0.004 & -0.028 & 0.029 & 0.973 & 0.332 & 0.006 & 0.036 & 0.006 \\
\hline RDST & 0.236 & -0.001 & 0.067 & 0.056 & 0.461 & 0.020 & 0.057 & $<0.001$ \\
\hline SCT (sec) & -0.084 & -0.409 & 0.204 & 0.505 & -0.271 & -0.460 & -0.028 & 0.028 \\
\hline SCT, no. of errors & -0.208 & -0.018 & 0.001 & 0.094 & -0.576 & -0.067 & -0.032 & $<0.001$ \\
\hline
\end{tabular}

A $P$-value $<0.05$ considered statistically significant.

BDNF, brain-derived neurotrophic factor; Cl, confidence interval; LB, lower bound; UB, upper bound; MMSE, mini-mental state examination; ERPs-P300, event-related potentials P300; ART, auditory reaction time; VRT, visual reaction time; Mini-CAQ, mini-cognitive assessment questionnaire; FDST, forward digit span test; RDST, reverse digit span test; SCT, symbol cancellation test. 
than in males in both the non-obese and the obese groups, but the difference was not statistically significant (Supplementary Table 1). Supplementary Fig. 1 shows a scatter plot of the correlation of obesity indices (BMI, WC, TC, and TG) with the BDNF levels in the participants. The correlation of cognitive parameters (MMSE, ERPs-P300 latency, Mini-CAQ and ART) with the BDNF levels in the participants are shown in Supplementary Fig. 2.

\section{DISCUSSION}

In the present study, we have assessed anthropometry, cognition parameters (ERPs-P300, ART, VRT, MMSE, Mini-CAQ SCT, STMT, FDST, and RDST), and biochemical parameters (glycemic profile, lipid profile, and BDNF) in young South-Indian adult population with obesity and an apparently healthy non-obese group. Both groups were comparable in terms of mean age. There was no significant difference due to sex in both groups in the present study. Sex as a confounder was nullified in the study.

Anthropometric and body composition parameters like BMI, WHR, body fat $\%$, and dry lean weight $\%$ were higher while lean fat $\%$ was lower in the obese group compared to the non-obese group, which denoted the increased fat mass and decreased free fat mass in the obese population. We found decreased cognitive task performance and serum BDNF levels in the obese group compared to the non-obese group.

The decreased BDNF levels may be due to mutation of the BDNF gene or TrkB receptors in the obese. ${ }^{23,24}$ It is still uncertain whether individuals with mutations that are expected to change BDNF expression or function have significant alterations in serum BDNF or whether serum BDNF will be useful to identify individuals anticipated to have resistance to the actions of BDNF at its receptor. Our findings were in contrast with those of Monteleone et al., ${ }^{23}$ which recorded increased serum BDNF levels in people with high BMI and which positively correlated BDNF levels with BMI and weight. This could be due to the differences in ethnicity, population age group, study design, methodology, and the sample size. Thus, to generalize these findings to the entire population with obesity, validation can be done with a large sample size with multicentric studies. However, we found higher BDNF levels in females than males in both the non-obese and obese groups, but we did not find any significant difference between them. Our findings were in line with those of Yarube et al. ${ }^{25}$

We found significant differences in the ERPs-P300 latency and amplitude between the non-obese and obese groups. ERPs-P300 latency and amplitude were negatively and positively associated with the BDNF levels in the obese population, respectively. This delayed latency indicated the impairment of attention, memory, and executive function of an individual. Our findings were supported by those of Mandelman and Grigorenko, ${ }^{24}$ which showed that the ERPs-P300 cognitive process is affected by the BDNF polymorphism, resulting in neurodegenerative disorders. Also, both the decreased amplitude and prolonged latency of ERPs-P300 were associated with insulin resistance in our study population, with the obese group showing the diminishing activity of neural circuits of sensory and cognitive processing. Our findings agreed with those of Tascilar et al. ${ }^{26}$

The reaction times of both ART and VRT were significantly reduced in the obese population in the present study. The VRT was comparatively more delayed than the ART. The increased delay of VRT relative to ART could be due to the chemical process of the visual pathway and the many collaterals throughout the pathway. ${ }^{27}$ Both ART and VRT reflect essential processes of central and peripheral pathways. These latencies are formed as the end response of a stimulation formed by the reflex arc, which involves both peripheral and central processing systems. ${ }^{27}$ Our findings were similar to the findings of Deore et al. ${ }^{28}$ However, in contrast with our findings, Shenvi et al., ${ }^{27}$ found that the ART latency was delayed more than the VRT, stating that the auditory pathway was more of a polysynaptic pathway than a visual pathway.

From the present study, we observed that the MMSE score and cognitive assessment scores were significantly reduced in the obesity group, and they were positively associated with the BDNF level. These results indicated that BDNF can be used as a biomarker for cognitive impairment in the obese population. Moreover, the central BDNF levels could be parallel to that of the peripheral BDNF levels. ${ }^{25} \mathrm{BDNF}$ has both neura ${ }^{29}$ and non-neural ${ }^{30}$ origins and can help in the development of neural circuits in the brain ${ }^{31}$ and cognitive function via neuroplasticity ${ }^{32}$ after crossing the blood-brain barrier. ${ }^{25}$ These neural circuits help in basic learning, memory, and other cognitive functions. Our results were consistent with the findings of Alfimova et al., ${ }^{33}$ showed that high serum BDNF levels in 
healthy adults were associated with better cognitive performance.

The time taken to perform and the number of errors in the SCT was significantly associated with lower BDNF levels in the obese population in our study. This association implied that the high BMI altered executive functions because of the disruption of frontal and subcortical circuits. ${ }^{34}$ The high BMI level could result in an impairment of decision-making, inhibition of response, and cognitive flexibility. ${ }^{35}$ Obesity was also shown to affect the frontal lobe structures and lowered the cortical thickness, in turn affecting executive decision making in the obese population, which consequently made more errors during the cognitive performance tasks. ${ }^{36}$ Our findings concurred with those of Marqués-Iturria et al., ${ }^{36}$ who found a correlation of lower BDNF levels with obesity, resulting in lower cortical thickness, which showed more errors in cognitive performance tasks.

We found a negative correlation between the BDNF and the glycemic profile (FBG, HbAlc, fasting insulin, and HOMA-IR). Reduced BDNF levels impaired the glucose metabolism, which could cause the obese population to be prone to diabetes mellitus in the future. This result was supported by the increased fasting serum insulin and HOMA-IR in the present study. These findings agreed with those of Tonra et al., ${ }^{37}$ which showed that a lower level of BDNF was seen in type 2 diabetes mellitus. Our findings concurred with the previous findings of $\mathrm{Das}^{38}$ that BDNF administration once or twice a week controlled the blood glucose in a diabetic mice model. We could conclude that BDNF has an anti-glycemic effect on glucose metabolism, but further studies should be performed to establish to see the same effect in humans before the treatment is implemented in clinical settings.

Our study shows that TC, TG, and LDL-C are negatively associated whereas HDL-C is positively associated with the serum BDNF levels in the obese group. These high BDNF levels are expressed via $\mathrm{BDNF} / \mathrm{TrkB}$ at the feeding center and hypothalamus in the energy homeostasis in animals. ${ }^{39}$ The deletion of adipose tissue and hypothalamic BDNF/TrkB axis in the experimental animals results in hyperphagia, leading to obesity and its related morbidities. In contrast to our results, Pelleymounter et al. ${ }^{40}$ have found a positive association of TC, TG, and LDL-C with BDNF. The role of BDNF is still controversial and inconsistent. The strengths of the present study are that we assessed a variety of cognitive domains, such as attention, memory, and executive function, by using standardized techniques. Further, the mean age difference is very narrow within the group and between the groups, which eliminates the effect of age and its related cognitive decline in both groups.

To conclude, the present study has been conducted in an obese population and as well as apparently healthy non-obese young adults to avoid other influences on the parameters studied. BDNF is negatively associated with cognition in the present study. This is confirmed by delayed ERPs-P300 latency, VRT, ART, SCT, and decreased cognitive scores like MMSE, mini-CAQ and DST in the obese population. Lower BDNF is associated with insulin resistance and hyperphagia, resulting in the accumulation of fat in the body. These results are evident by the increased HOMA-IR and lipid profile in the obese population. All of these changes together may contribute to an alteration of the process of neuroplasticity in the obese population. The findings from the present study suggest that reduced circulating BDNF levels are strongly associated with decline in cognitive parameters such as attention, memory, and executive functions in obese South Indian adults. To generalize our findings to the entire population with obesity, a large sample size with multicentric studies may be required. Because these results were found in South Indian population with obesity, we cannot generalize our findings to the entire population with obesity. To validate our findings a large sample size with multicentric studies is required.

\section{CONFLICTS OF INTEREST}

The authors declare no conflict of interest.

\section{ACKNOWLEDGMENTS}

Authors are thankful to all the study participants, who have given their consent to be in the study. We also thank the JIPMER Institute research funding (JIP/Res/Intramural/Phs1/2018-19/99) for extending financial support for our project.

\section{AUTHOR CONTRIBUTIONS}

Study concept and design: all authors; acquisition of data: RBK; analysis and interpretation of data: RBK, GSG, and KS; drafting of 
the manuscript: RBK, GSG, and KS; critical revision of the manuscript: RBK, GSG, KS, JPS, and ZB; statistical analysis: RBK and KS; obtaining funding: GSG and RBK; administrative, technical, or material support: GSG and KS; and study supervision: GSG.

\section{SUPPLEMENTARY MATERIALS}

Supplementary Table 1 and Figures 1 and 2 can be found via https://doi.org/10.7570/jomes20107.

\section{REFERENCES}

1. World Health Organization. Obesity and overweight [Internet]. Geneva: World Health Organization; 2020 [cited 2021 Mar 31]. Available from: http://www.who.int/mediacentre/ factsheets/fs311/en/

2. Bray GA. Medical consequences of obesity. J Clin Endocrinol Metab 2004;89:2583-9.

3. Wang C, Chan JS, Ren L, Yan JH. Obesity reduces cognitive and motor functions across the lifespan. Neural Plast 2016; 2016:2473081.

4. Australian Bureau of Statistics. Australian health survey: first results, 2011-12. Belconnen: Australian Bureau of Statistics; 2012.

5. Lebrun B, Bariohay B, Moyse E, Jean A. Brain-derived neurotrophic factor (BDNF) and food intake regulation: a minireview. Auton Neurosci 2006;126-127:30-8.

6. Yeo GS, Connie Hung CC, Rochford J, Keogh J, Gray J, Sivaramakrishnan $S$, et al. A de novo mutation affecting human TrkB associated with severe obesity and developmental delay. Nat Neurosci 2004;7:1187-9.

7. Cao L, Lin EJ, Cahill MC, Wang C, Liu X, During MJ. Molecular therapy of obesity and diabetes by a physiological autoregulatory approach. Nat Med 2009;15:447-54.

8. Slusher AL, Whitehurst M, Zoeller RF, Mock JT, Maharaj A, Huang CJ. Brain-derived neurotrophic factor and substrate utilization following acute aerobic exercise in obese individuals. J Neuroendocrinol 2015;27:370-6.

9. Motamedi S, Karimi I, Jafari F. The interrelationship of metabolic syndrome and neurodegenerative diseases with focus on brain-derived neurotrophic factor (BDNF): kill two birds with one stone. Metab Brain Dis 2017;32:651-65.

10. Hinderberger P, Rullmann M, Drabe M, Luthardt J, Becker GA, Blüher M, et al. The effect of serum BDNF levels on central serotonin transporter availability in obese versus nonobese adults: a [(11)C]DASB positron emission tomography study. Neuropharmacology 2016;110:530-6.

11. World Health Organization. The Asia-Pacific perspective: redefining obesity and its treatment. Geneva: World Health Organization; 2000.

12. Choi SH, Ku EJ, Hong ES, Lim S, Kim KW, Moon JH, et al. High serum adiponectin concentration and low body mass index are significantly associated with increased all-cause and cardiovascular mortality in an elderly cohort, "adiponectin paradox": the Korean Longitudinal Study on Health and Aging (KLoSHA). Int J Cardiol 2015;183:91-7.

13. Kumar N, Sood S, Singh M, Beena, Sakshi. Effect of acute moderate exercise on cognitive event-related potentials n100, p200, n200, and interpeak latencies. Indian J Psychol Med 2010;32:131-5.

14. Andreadou E, Mitrakou A, Constantinides VC, Triantafyllou N. Auditory P300 event-related potentials in patients with type 2 diabetes mellitus. J Diabetes Res Clin Metab 2012;1:1.

15. Sharma VK, Subramanian SK, Arunachalam V, Radhakrishnan K, Ramamurthy S, Ravindran BS. Auditory and visual reaction times in school going adolescents: effect of structured and unstructured physical training: a randomized control trial. Int J Adolesc Med Health 2015;29:20150060.

16. Folstein MF, Folstein SE, McHugh PR. "Mini-mental state": a practical method for grading the cognitive state of patients for the clinician. J Psychiatr Res 1975;12:189-98.

17. Cordell CB, Borson S, Boustani M, Chodosh J, Reuben D, Verghese J, et al. Alzheimer's Association recommendations for operationalizing the detection of cognitive impairment during the Medicare Annual Wellness Visit in a primary care setting. Alzheimers Dement 2013;9:141-50.

18. Brucki SM, Nitrini R. Cancellation task in very low educated people. Arch Clin Neuropsychol 2008;23:139-47.

19. Monaco M, Costa A, Caltagirone C, Carlesimo GA. Forward and backward span for verbal and visuo-spatial data: standard- 
ization and normative data from an Italian adult population. Neurol Sci 2013;34:749-54.

20. Wallace TM, Levy JC, Matthews DR. Use and abuse of HOMA modeling. Diabetes Care 2004;27:1487-95.

21. Friedewald WT, Levy RI, Fredrickson DS. Estimation of the concentration of low-density lipoprotein cholesterol in plasma, without use of the preparative ultracentrifuge. Clin Chem 1972;18:499-502.

22. Dobiásová M, Frohlich J. The plasma parameter log (TG/ HDL-C) as an atherogenic index: correlation with lipoprotein particle size and esterification rate in apoB-lipoprotein-depleted plasma (FER(HDL)). Clin Biochem 2001;34:583-8.

23. Monteleone P, Tortorella A, Martiadis V, Serritella C, Fuschino A, Maj M. Opposite changes in the serum brain-derived neurotrophic factor in anorexia nervosa and obesity. Psychosom Med 2004;66:744-8.

24. Mandelman SD, Grigorenko EL. BDNF Val66Met and cognition: all, none, or some? A meta-analysis of the genetic association. Genes Brain Behav 2012;11:127-36.

25. Yarube IU, Hassan TM, Ahmad RY, Umar LM, Musa BM, Ibrahim SM. Association between cognition and peripheral brain-derived neurotrophic factor in a sample of normal adults in Kano, Nigeria. Niger J Basic Clin Sci 2019;16:55-9.

26. Tascilar ME, Turkkahraman D, Oz O, Yucel M, Taskesen M, Eker I, et al. P300 auditory event-related potentials in children with obesity: is childhood obesity related to impairment in cognitive functions? Pediatr Diabetes 2011;12:589-95.

27. Shenvi D, Balasubramanian P. A comparative study of visual and auditory reaction times in males and females. Indian J Physiol Pharmacol 1994;38:229-31.

28. Deore DN, Surwase SP, Masroor S, Khan ST, Kathore V. A cross sectional study on the relationship between the body mass index (BMI) and the audiovisual reaction time (ART). J Clin Diagn Res 2012;6:1466-8.

29. Béjot Y, Mossiat C, Giroud M, Prigent-Tessier A, Marie C. Circulating and brain BDNF levels in stroke rats. Relevance to clinical studies. PLoS One 2011;6:e29405.

30. Lu B, Nagappan G, Lu Y. BDNF and synaptic plasticity, cognitive function, and dysfunction. Handb Exp Pharmacol 2014; 220:223-50.

31. Yu H, Chen ZY. The role of BDNF in depression on the basis of its location in the neural circuitry. Acta Pharmacol Sin 2011;32:3-11.

32. Leal G, Afonso PM, Salazar IL, Duarte CB. Regulation of hippocampal synaptic plasticity by BDNF. Brain Res 2015; 1621:82-101.

33. Alfimova MV, Korovaitseva GI, Lezheiko TV, Golimbet VE. Effect of BDNF Val66Met polymorphism on normal variability of executive functions. Bull Exp Biol Med 2012;152:606-9.

34. Stanek KM, Strain G, Devlin M, Cohen R, Paul R, Crosby $\mathrm{RD}$, et al. Body mass index and neurocognitive functioning across the adult lifespan. Neuropsychology 2013;27:141-51.

35. Siervo M, Arnold R, Wells JC, Tagliabue A, Colantuoni A, Albanese E, et al. Intentional weight loss in overweight and obese individuals and cognitive function: a systematic review and meta-analysis. Obes Rev 2011;12:968-83.

36. Marqués-Iturria I, Garolera M, Pueyo R, Segura B, Hernan I, García-García I, et al. The interaction effect between BDNF val66met polymorphism and obesity on executive functions and frontal structure. Am J Med Genet B Neuropsychiatr Genet 2014;165:245-53.

37. Tonra JR, Ono M, Liu X, Garcia K, Jackson C, Yancopoulos GD, et al. Brain-derived neurotrophic factor improves blood glucose control and alleviates fasting hyperglycemia in C57BLKSLepr(db)/lepr(db) mice. Diabetes 1999;48:588-94.

38. Das UN. Obesity: genes, brain, gut, and environment. Nutrition 2010;26:459-73.

39. Schwartz MW, Woods SC, Porte D Jr, Seeley RJ, Baskin DG. Central nervous system control of food intake. Nature 2000; 404:661-71.

40. Pelleymounter MA, Cullen MJ, Wellman CL. Characteristics of BDNF-induced weight loss. Exp Neurol 1995;131:229-38. 